\title{
Name Font Design of the City Commercial Complex Reflecting the City's Image
}

\author{
Yan Jiaxiang \\ Zhejiang University of Technology, Zhijiang College
}

Keywords: commercial complex, design, font, industrial design

\begin{abstract}
Starting from the identification system elements of public facilities modeling system, city identification guidance system and urban outdoor advertisement, this paper analyzes the technical and artistic level of the urban brand visual image recognition system. By drawing on the design principle of the enterprise image and the idea of urban construction in the first place at home and abroad, the vision of the brand vision is made. The image recognition system construction method makes a theoretical summary, and provides suggestions and references for other urban construction with regional characteristics.
\end{abstract}

\section{Introduction}

Outdoor advertising is usually the most important place in the street, so the design of its form, color, content, volume, cultural connotation and other aspects of the design form the focus of visual attraction in the street space. Outdoor advertising should not only achieve the most significant interests of its business, but also have to coordinate with other landscape elements in urban streets, such as urban architecture and greening, so that it can be used in all aspects such as color, material or location, and the overall street landscape of the city. It is able to develop in a harmonious way. Therefore, by maximizing the dual role of the art of urban outdoor advertising and its cultural carrier, it not only improves the public's sense of identity and identifiability to the urban street space, but also embodies the characteristics of the street landscape, and makes the overall landscape of the city street and the outdoors wide open to each other.

There are different requirements for outdoor advertisements for special districts in cities. Taking Tokyo as an example, the zoning of advertising is determined according to the zoning requirements of the landscape planning formulated by the city. Including historical and cultural preservation areas (a total of 7 pieces), waterscape formation special area (a total of 54 pieces), Motian district (Tokyo characteristic landscape area, a total of 2 pieces). In this particular area, the requirements for outdoor advertising are more strict, and detailed regulations are made on how to keep the requirements of the landscape planning, the permission and prohibition of advertising, the color of the advertisement and the use of the light source.

The rapid development of the modernization of science and technology, in addition to the convenience of people's daily life, is also "upgrading" a new form of outdoor advertising incorporating modern scientific and technological means to create a new form of advertising media, which has created a new type of advertising, such as electronic billboards, touch interactive screens, high security, great acceptance and promotion of urban color. Media.

\section{Ad Font Design}

\subsection{Design Thought}

Ideological and unitary layout is not an end in itself. Design is a means to better disseminate customer information. Designers often intoxicated with their personal style and the font and graphics that are not consistent with the theme, which is often the main reason for the mediocre failure of design. For a successful layout, we must first define the purpose of the client and thoroughly understand, observe, study and design all aspects. A brief consultation is the beginning of a good design. Layout can not be separated from content, but also reflects the theme of the content, so as to enhance the reader's attention and understanding. Only when we have a clear 
theme and a clear view can we achieve the ultimate goal of layout. The theme is bright and outstanding, which is the best embodiment of design thought. Graphic art can only be contacted with readers in limited space, which requires that the layout must be simple and concise. People have long ignored the cramming and intricate layout of the past. In fact, the emphasis on simplicity and simplicity is not monotonous and simple, but the concentration of information and the refined expression of the content, which is based on a novel and unique artistic conception. Therefore, the simplification of the layout includes not only the planning and refining of the content of the appeal, but also the composition skills of the layout. Focusing on the product itself and flooding the entire layout, it is striking and striking.

In order to make the layout better serve the content of the page, it is very important to seek a reasonable layout of the layout, and it is also the embodiment of the best appeal. Conception is the first step in design, and it is also the thinking activity in design works. After the theme is clear, layout and form of layout become the core of the art of layout design, and it is also an arduous creation process. How to achieve new ideas, shape beauty, change and unity, and have aesthetic taste, it depends on the conservation of designer culture. Therefore, page composition is a comprehensive examination of the designer's ideological realm, artistic accomplishment and technical knowledge. The decorative factors of the layout are the composition and arrangement of words, graphics and colors through points, lines and surfaces, and use exaggerated, figurative and symbolic techniques to embody visual effects. It not only beautifies the layout, but also improves the function of conveying information. Decoration is constructed by the use of aesthetic features. Different types of layout information, with different forms of decoration, not only to exclude other, highlight the role of the layout of information, but also to enable readers to enjoy the beauty of the beauty.

\subsection{Character Design Features}

(1) A large number of print advertising media are available for indoor or small scale communication. The outdoor light box advertisement displays the contents of advertisements through doorways, announcements (publicity columns) and vertical pole boxes. It is much more attractive than other print advertisements with large illustrations and large fonts. (2) hyperopia, the function of outdoor light box advertising, is through the natural light (day), auxiliary light (night) two forms, to the outdoors people, long distance people to convey information. The far sighted effect of advertising works is very conducive to the fast paced, efficient and fast-moving people in modern society. (3) a wide range of content, in the public class of transportation, transportation, safety, welfare, savings, insurance, tax and other aspects; in the commercial products, enterprises, tourism, service and other aspects, culture, education, art and other aspects of the culture and education can play a wide role. (4) concurrently, there are various forms of display, with the function of both text and color. The advertising language designer has everything from product trademark, name, object photo, color, enterprise intention to culture, economy, custom, belief and concept. Through conception and unique creativity, we must firmly grasp the "ring" that induces consumers' desire to purchase, and achieve the purpose of advertising with the heterogeneity of visual communication. (5) fixation and complexity, no matter what form the outdoor light box advertisement is used, the advertisement layout design has its fixed requirements in a certain range and location. As a semi permanent display device, its basic structure is more complex than other advertising forms. It includes frame, surface material, pattern printing layer, wind proof, rain and snow structure, and lighting facilities at night, making the single copy cost higher than other types of advertising. Outdoor advertising uses its application places on roads and streets, as well as shadow (Drama), exhibition (sale), commercial downtown, station, airport, dock, park and other public places. It is called "semi permanent" street art in foreign countries. Outdoor light box advertisements are different from other types of advertisements. 


\section{Design of a Small Business Complex}

\subsection{Scientific and Reasonable Integrated Design}

The commercial complex contains a wealth of functions, such as hotel functional buildings, office functional buildings, catering, entertainment facilities, residential functional buildings, etc. The main characteristic of the commercial complex is to organize the different functions and the inner relations of the interval scientifically and scientifically, and create different types of combinations, among which the core types are as follows: first, the functional complementary type, that is, the organic whole which is formed by complementary design of some different work energy intervals. For example, catering matching is a powerful service to the needs of office workers. Second, function series, that is, by combining some functional areas with continuity and interoperability. For example, there are some early education institutions and children's entertainment establishments in the building group, which can also drive other store businesses. Third, functional competition, that is, through the diversity of services and market demand, set up a number of similar functional areas in parallel to promote its agglomeration effect. Of course, the actual commercial complex is the result of flexibly applying these types of system combinations according to market demand.

\subsection{Perfect the Reasonable Traffic System}

At present, whether big city or small city traffic problem is a headache for everyone when they travel. Whether the road is congested and whether the parking design organization is in place directly affects the efficiency of the commercial operation, and at the same time, it concerns the efficiency of the building in the interval. The most important factor that should be considered for the design of parking organization is the different parking mode chosen by the customers. This is a comprehensive problem. Most customers like to stop on the ground so that they can be very convenient to leave after the consumption is good, but this involves the problems of land use and economy. Under normal circumstances, due to the high land price, the commercial complex's ground space has no way to meet the parking demand. At that time, underground parking will be chosen. However, the cost of underground parking is much higher than that of parking on the ground, and at the same time, the access is not very convenient. Therefore, the design of underground parking access is reasonable. To improve parking organization design, we must increase parking space to a maximum extent through scientific and rational planning.

Then, the design of the traffic system should take full consideration of the factors of the traffic situation and the characteristics of the terrain, and choose the organization form of the traffic system in the commercial complex in detail and carefully. For example, the setting of the entrance and exit, the guidance of the traffic flow are very important. If we want to consider the flow of people and traffic as the basic market demand, the organization form of the traffic system can be divided into two forms: human and car mixing and human and vehicle shunting. First, the model of human and car mixing has a certain degree of flexibility, the accessibility of consumption is better, but there are a lot of interference. Unsafe factors. Therefore, we must make use of the tortuous and rough materials in the planning to effectively limit the speed. Secondly, the mode of human and car shunting which is independent of human flow and traffic flow has the advantages of clear streamline and little interference, but the long streamline hinders the traffic flow while ensuring the convenience of people. Each of the two ways has advantages and disadvantages. It is necessary to balance the traffic organization form as far as possible, which is convenient for parking and shopping.

\subsection{Design of Functional Space}

The interior function space design of commercial complex is very important. Reasonable function allocation and layout can effectively connect each functional space in series. For example, light catering can be set up on each floor, and heavy catering is set at a certain level. This enables customers to drink coffee, buy cakes and so on while shopping. At the same time, you can have a 
purpose to eat at a big restaurant in the mall.

\section{LOGO Font Design for Business Complex}

In order to make the layout better serve the content of the page, it is very important to seek a reasonable layout of the layout, and it is also the embodiment of the best appeal. Conception is the first step in design, and it is also the thinking activity in design works. After the theme is clear, layout and form of layout become the core of the art of layout design, and it is also an arduous creation process. How to achieve new ideas, shape beauty, change and unity, and have aesthetic taste, it depends on the conservation of designer culture. Therefore, page composition is a comprehensive examination of the designer's ideological realm, artistic accomplishment and technical knowledge. The decorative factors of the layout are the composition and arrangement of words, graphics and colors through points, lines and surfaces, and use exaggerated, figurative and symbolic techniques to embody visual effects. It not only beautifies the layout, but also improves the function of conveying information. Decoration is constructed by the use of aesthetic features. Different types of layout information, with different forms of decoration, not only to exclude other, highlight the role of the layout of information, but also to enable readers to enjoy the beauty of the beauty.

The interest in typesetting design mainly refers to the interest of form. This is a lively language of layout. If there are not many wonderful contents in the layout, it is necessary to create interest to win. This is also the role of artistic means in conception. The layout is full of interest, which makes media information more powerful and more effective. Interest can be obtained by means of expression, humor and lyric. The principle of originality is in essence the principle of individualized characteristics. Distinctive personality is the creative soul of typesetting design. Just imagine that a layout is much the same as simplification and conceptualization. Not to talk about winning. Therefore, to dare to think, dare to be unique, dare to be unique, in the design of typesetting more personality and less common, more ingenuity and less general, to win the favor of the consumer.

\section{Conclusion}

Starting from the identification system elements of public facilities modeling system, city identification guidance system and urban outdoor advertisement, this paper analyzes the technical and artistic level of the urban brand visual image recognition system. By drawing on the design principle of the enterprise image and the advanced urban construction ideas at home and abroad, the visual image of the brand is made. This paper makes a theoretical summary of the construction method of the identification system, and provides suggestions and references for other urban construction with regional characteristics.

\section{References}

[1] Sun X, Xue Y, Liang C, Wang T, Zhe W, Sun G, Li X, Li X, Liu G. Histamine Induces Bovine Rumen Epithelial Cell Inflammatory Response via NF-кB Pathway[J]. Cellular Physiology \& Biochemistry, 2017, 42(3):1109-1119.

[2] Du, X., Y. Zhu, Z. Peng, Y. Cui, Q. Zhang, Z. Shi, Y. Guan, X. Sha, T. Shen, Y. Yang, X. Li, Z. Wang, X. Li, and G. Liu. High concentrations of fatty acids and beta-hydroxybutyrate impair the growth hormone-mediated hepatic JAK2-STAT5 pathway in clinically ketotic cows[J]. J Dairy Sci, 2018, 0302(18):30029-8

[3] Yijiu Zhao, Li Wang, Houjun Wang, and Changjian Liu. Minimum Rate Sampling and Spectrum Blind Reconstruction in Random Equivalent Sampling[J]. Circuits Systems and Signal Processing, 2015, 34(8):2667-2680.

[4] Arunkumar, N., Ramkumar, K., Venkatraman, V., Abdulhay, E., Fernandes, S. L., Kadry, S., \& Segal, S. Classification of focal and non focal EEG using entropies[J]. Pattern Recognition Letters, 
2017,94:112-117

[5] Malarkodi, M.P., Arunkumar, N., Venkataraman, V. Gabor wavelet based approach for face recognition [J]. International Journal of Applied Engineering Research, 2013, 8(15):1831-1840. 